\title{
Impact of a Combined Value-Based Insurance Design and Medication Therapy Management Program on Diabetes Medication Adherence
}

\author{
Alex Peaslee, PharmD; Marleen Wickizer, PharmD, AE-C; \\ Julie Olson, DNP, MS, RN, CQIA, CBE; and Robert Topp, PhD, RN
}

\begin{abstract}
BACKGROUND: Value-based insurance design (VBID) waives or reduces prescription copayments in order to decrease member cost barriers to refilling medications. Medication therapy management (MTM) is a member clinical intervention designed to reinforce members' knowledge of their medications, which addresses barriers to medication adherence. Both methods have been shown to increase adherence in members, particularly when used in combination. To date, studies of such combined programs have often been completed within integrated health systems but have rarely included control populations.
\end{abstract}

OBJECTIVE: To determine the effect of a combined VBID and MTM program on key medication adherence metrics among diabetic members of a large employer group in the Midwest.

METHODS: A retrospective pre/post longitudinal analysis of pharmacy claims data was performed for 77 participants in a combined VBID/MTM program and 77 eligible nonparticipants, matched by the baseline adherence metrics of proportion of days covered (PDC) and number of days without therapy, also known as gaps in therapy (GIT). Oral antidiabetic medication adherence and cost-related outcomes for all pharmacy claims were evaluated within and between groups over a 6-month period. Post hoc analyses were performed to investigate the effect of the intervention by gender and among a less adherent subpopulation of participants with a PDC of $<100 \%$ at baseline.

RESULTS: Introduction of the intervention resulted in a nonsignificant increase in PDC from $92.9 \%$ to $95.4 \%$, in contrast to a nonsignificant decrease from $92.8 \%$ to $91.7 \%$ in the comparison group. GIT underwent a nonsignificant decrease of 2.83 days during intervention, while nonsignificantly increasing 2.82 days in the comparators. Pharmacy claims costs paid by the plan per member per 6-month period significantly increased in the intervention group from $\$ 1,991.23$ to $\$ 3,092.74$, compared with a nonsignificant increase from $\$ 1,402.21$ to $\$ 1,645.68$ in the comparison group. Among the less-adherent subpopulation, PDC increased significantly after intervention from $84.7 \%$ to $93.1 \%$ compared with a nonsignificant increase from $84.6 \%$ to $89.0 \%$ among nonparticipants. A significant 10.69 -day decrease in GIT was also observed among nonadherent participants compared with a nonsignificant 3.59-day decrease among nonparticipants. Female participants experienced a significant PDC increase from $91.5 \%$ to $96.8 \%$ and a GIT decrease of 7.32 days, while male participants did not change significantly.

CONCLUSIONS: While statistically significant improvements to adherence were not observed among this population of members who were highly adherent at baseline, improvement trends and subgroup analyses demonstrated that the combined VBD/MTM program may have the potential to influence member behavior in employer groups. Larger, longer-term studies are needed to confirm this potential. Additional benefit may be realized by targeting members with lower adherence metrics at baseline and examining potential cost savings associated with medical outcomes.

J Manag Care Spec Pharm. 2016;22(11):1303-09

Copyright $\odot 2016$, Academy of Managed Care Pharmacy. All rights reserved.

\section{What is already known about this subject}

Value-based insurance design (VBID) and medication therapy management (MTM) have been shown to improve medication adherence individually and synergistically.

Improved adherence to diabetic medications results in improved clinical outcomes and decreased overall health care costs.

\section{What this study adds}

A combined VBID and MTM program led to trends toward improvement in real-world proportion of days covered (PDC) and gaps in therapy (GIT) within a large employer group compared with a similar population of nonparticipants.

Significant improvements in PDC and GIT were observed among members with suboptimal adherence at baseline after combined VBID and MTM intervention.

PDC and GIT for female members improved significantly in response to a combined VBID/MTM program, while metrics for male members did not improve.

T alue-based insurance design (VBID) is a method of reducing barriers to the access of medications with significant clinical value by waiving or reducing member copayments. Medication therapy management (MTM) is a clinical intervention in which a health care provider, commonly a pharmacist, provides individualized assessment and education regarding medication therapies and disease management. VBID and MTM have been emerging topics in pharmacy benefit management in recent years. With increasing health care costs and increasing pressure to maximize clinical outcomes while controlling costs, these programs are believed to address suboptimal medication adherence, which in turn improves medication outcomes and ultimately reduces total health care costs.

One of the primary barriers to optimal medication adherence is member prescription costs. According to a review of 24 studies focused on medication adherence, medication copayments had the strongest association with nonadherence of all factors studied, with nonadherence rates doubling with the introduction of a $\$ 10$ copayment. ${ }^{1}$ Additional research shows that decreasing copayments may decrease nonadherence rates. ${ }^{2}$ Furthermore, a survey of Medicare beneficiaries found that cost was a driving factor behind more than half of all members failing to fill prescriptions. ${ }^{3}$ 
VBID attempts to address this barrier to adherence for medications considered especially valuable. Valuable medications may be identified individually or grouped together as medications treating specific diseases of high therapeutic value, such as hypertension, congestive heart failure, or diabetes. Lowering copayments for high-value medications in turn encourages adherence. ${ }^{4}$ A review of 13 studies of VBID programs found consistent improvements in adherence. ${ }^{5}$ In 1 study, VBID significantly decreased cost-related nonadherence from $41 \%$ to $17.5 \%$ and led to a high degree of self-reported satisfaction with the program. ${ }^{6}$

Another significant barrier to medication adherence is member health literacy. Members were found to be twice as adherent when they had an adequate understanding of their disease state and the purpose of the medications they were prescribed. ${ }^{1}$ MTM, in which a pharmacist performs a comprehensive review of a members' medication regimen, including assessments of efficacy, appropriateness of medication, and education of the member in an outpatient setting, provides 1 method of addressing this barrier. ${ }^{7}$ Such MTM programs can significantly lower chronic disease state medication discontinuation rates. ${ }^{8,9}$

Programs that increase medication adherence may decrease overall health care spending. For example, medication nonadherence has been shown to significantly increase all-cause hospitalizations. ${ }^{10}$ Nonadherence may be a contributing factor to poorly managed chronic disease. Poorly managed chronic diseases may require additional medication use to address symptoms resulting from poor control, further increasing health care spending. ${ }^{7}$ The Asheville Project demonstrated that a comprehensive MTM program correlates with increased medication use and decreased overall health care spending. ${ }^{9}$ A study of VBID found that the increased drug costs of the program were entirely offset by savings in total health care expenses. ${ }^{11}$ Additionally, the U.S. Congressional Budget Office has projected that each $1 \%$ increase in medication adherence can result in $0.2 \%$ savings from the total medical expenditure. ${ }^{12}$ VBID and MTM programs may be even more beneficial in combination. Although the previously mentioned review of VBID programs found that increased medication adherence did not significantly lower total health care costs, a subset of that review found that VBID, when combined with MTM, did result in decreased total health care costs. ${ }^{5}$

The body of evidence for combination programs involving VBID and MTM is still in development. There are limited studies of such programs, and very few have comparator groups. One such study is the Cincinnati Pharmacy Coaching Program, which identified significant improvements to members' medication adherence and health outcomes relative to a comparator group. ${ }^{13}$ The purpose of the current study is to determine the effect of a combined VBID and MTM program on key medication adherence metrics among diabetic members of a large employer group in the Midwest. The degree of pharmacy benefit cost increases resulting from the program will also be presented to provide a benchmark for the program.

\section{Methods}

This study was a retrospective evaluation of a previously designed and ongoing intervention. Intervention participants were members of a large employer group located in the midwestern United States. Participation was limited to employees only, excluding family members and dependents. Employees with a recorded diagnosis of diabetes (International Classification of Diseases, Ninth Revision, Clinical Modification codes 250.xx, $357.2 x, 362.0 x, 366.41$, or $645.0 x)$ were identified through health plan data and received information about the VBID/ MTM program-designated the "diabetes management program"- and an invitation to participate through the mail. The program began on May 1, 2013, and allowed for rolling admission of invited participants at any time. As of August 2014, 197 diabetic employees had elected to participate in the program (Figure 1). This study was exempted from review by the Dean Foundation Institutional Review Board because the research involved the study of existing data with removal of all subject identifiers.

The diabetes management program consisted of a multifaceted approach. Participants received face-to-face MTM sessions from a network of trained outpatient pharmacists at least twice per year. In addition to a comprehensive medication review, these MTM sessions focused on the achievement of a blood pressure $<140 / 90 \mathrm{mmHg}$, an $\mathrm{LDL}<100 \mathrm{mg} / \mathrm{dL}$, an Alc $<8 \%$, tobacco cessation (if applicable), and appropriate use of aspirin. The program also incorporated VBID by waiving copayments for all preferred formulary diabetic medications and supplies for participating members. Member participation was further incentivized with a $\$ 50$ contribution to the Health Reimbursement Account of members upon the initial MTM visit and enrollment. Participants were also provided with an 8- to 12-week optional lifestyle coaching program at no cost and received an additional \$200 Health Reimbursement Account contribution upon completion of the lifestyle program.

Members were included for analysis if they had completed an enrollment form for the program after their first MTM visit between June 1, 2013, and November 30, 2013, and had at least 1 pharmacy claim for an oral antidiabetic medication (OAM; defined by Generic Product Identifier codes beginning in 2720, $2725,2755,2760$, or 2799) in the 6-month periods preceding and following the intervention start date. For practicality of analysis, the intervention start date was considered to be the first of the month immediately following the month of application (e.g., if an application was submitted June 17, 2013, the intervention start date was considered July 1, 2013). Members were excluded from analysis if they joined the plan within 6 months before the intervention start date or left the plan within 6 months after the intervention start date. 


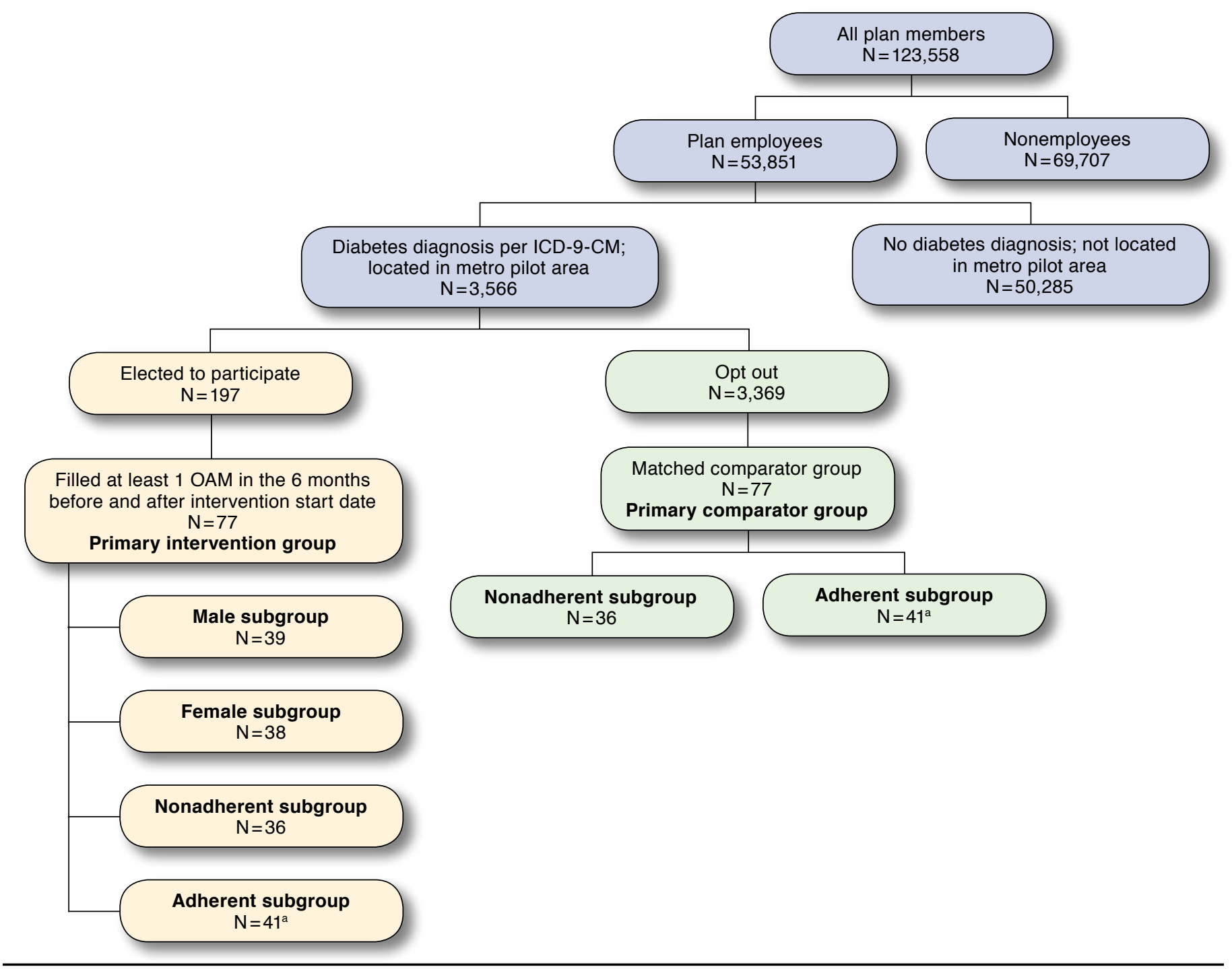

Note: Gender and adherence subgroups are independent of each other.

aProvided for clarity; not used for any analyses.

ICD-9-CM=International Classification of Diseases, Ninth Revision, Clinical Modification; OAM = oral antidiabetic medication.

Members included in the analysis were matched to a comparator group selected from a pool of nonparticipating plan members who had at least 1 OAM claim in the 6 months before and after the intervention start date of the matched participating member. Nonparticipating members had identical benefit structures with the exception of waived diabetic copayments. The standard benefit structure for both groups encouraged voluntary 90-day mail order use by reducing the 90-day copayment to the equivalent of two 30-day copayments.
Comparator members were selected by matching baseline characteristics to the participating population. Of the 4 baseline characteristics considered, the greatest weight was given to the proportion of days covered (PDC; equivalent to the ratio of days of medication supplied from the class over the number of days from first fill to the end of the time period) for OAMs, followed by the number of distinct OAMs filled, member age, and gaps in therapy (GIT; depicts the total number of days without medication over the study period) for OAMs. 


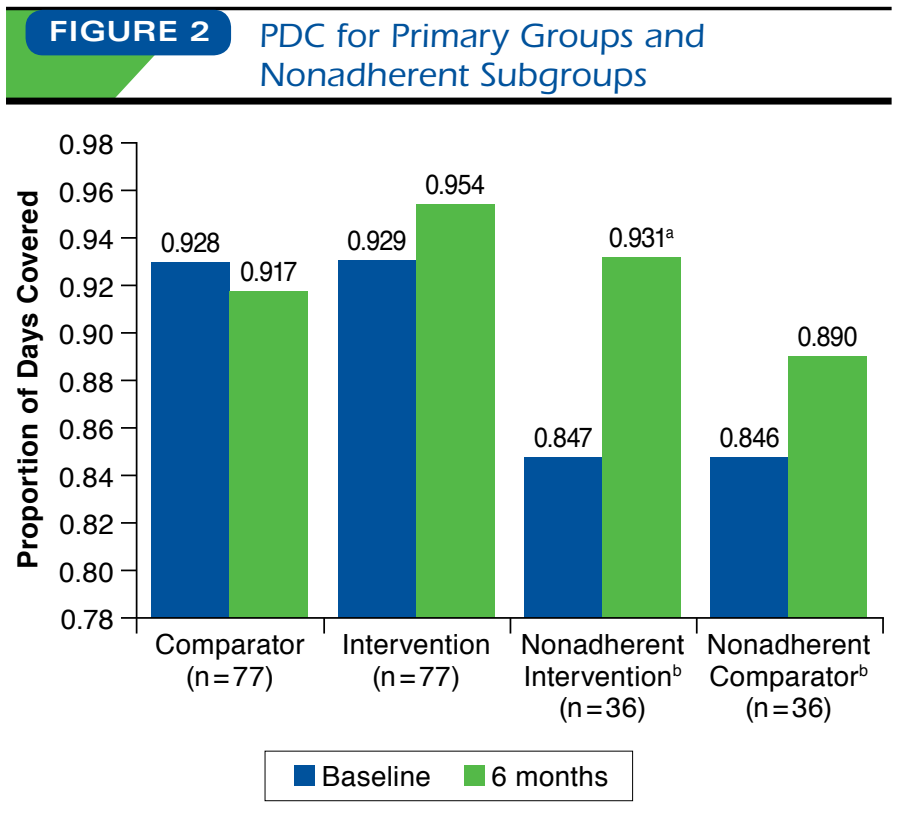

a $<0.05$ relative to nonadherent intervention group baseline.

${ }^{b}$ Nonadherent subgroup defined as having a baseline of $P D C<100 \%$.

$P D C=$ proportion of days covered.

Medication adherence metrics analyzed included the PDC and GIT for all OAMs, calculated by a proprietary internal program following the Pharmacy Quality Alliance methodology. The total plan-paid costs for all pharmacy claims, including OAMs, were collected within an internal claims database and analyzed. A retrospective pre/post longitudinal analysis was performed. All outcomes were measured within-groups (comparing the initial 6 months after intervention start date to the 6 months immediately preceding the intervention start date), as well as between-groups (comparing the intervention and comparator groups over the 6 months following the intervention start date).

A secondary post hoc analysis was performed based on preliminary observations to measure the within-subject impact of intervention on PDC and GIT between genders. Additionally, the within- and between-subjects effect of intervention on PDC and GIT was analyzed on a subset of members who had a PDC less than $100 \%$ at baseline, referred to as the nonadherent subpopulation.

\section{Statistical Analysis}

Member records, including baseline adherence metrics and plan-paid pharmacy costs, were entered into an SPSS spreadsheet (SPSS, Chicago, IL) and were determined to exhibit no outliers and met the assumptions of parametric statistics. Three separate repeated measures analyses of variance (ANOVAs) were calculated on the outcomes of PDC and GIT, comparing differences in these outcomes with and between study groups over the duration of the study. Significant main effects of the R-ANOVA were further examined through Tukey least significant post hoc comparisons to determine differences between group means. The initial analysis involved comparing the groups that did and did not receive the intervention. Following this analysis, the subgroup of nonadherent members from each of the study groups were identified and compared using the same R-ANOVA approach. Finally, only members who received the intervention were grouped by gender and compared with PDC and GIT over the duration of the study using the same R-ANOVA analysis and post hoc comparisons. All analyses set $P<0.05$ as the level of statistical significance a priori.

\section{Results}

Of the 197 members who elected to participate in the diabetes management program, 77 members met the inclusion and exclusion criteria. Of the 77 members, 39 were male and 38 were female. The nonadherent subpopulation consisted of 72 members: 36 members who received the intervention and 36 members from the comparison group.

\section{Proportion of Days Covered}

Intervention members exhibited a nonsignificant increase in PDC from $92.9 \%$ to $95.4 \%$. The PDC in the comparator group decreased nonsignificantly from $92.8 \%$ to $91.7 \%$. When considering the nonadherent subpopulation alone, the intervention resulted in a statistically significant increase in PDC from $84.7 \%$ to $93.1 \%(P<0.05)$, while the nonadherent comparison members exhibited a nonsignificant improvement in their PDCs from $84.6 \%$ to $89.0 \%$ (Figure 2).

\section{Gaps in Therapy}

Members who received the intervention exhibited a nonsignificant decrease of 2.83 days in their GIT. The comparison group demonstrated a nonsignificant increase of 2.82 days in their GIT. Among the nonadherent subpopulation, the intervention significantly decreased the GIT by 10.69 days $(P<0.05)$, while the comparison group experienced a nonsignificant 3.59-day decrease in GIT (Figure 3).

\section{Impact by Gender}

The 38 females who participated in the intervention experienced a statistically significant increase in PDC from 91.5\% to 96.8\% $(P<0.05)$, while the males in this group did not change on this variable (Figure 4). Female participants also achieved a statistically significant decrease in their GIT from 11.37 days to 4.05 days $(P<0.05)$, compared with the nonsignificant increase of 1.54 days exhibited by male participants (Figure 5).

\section{Plan-Paid Costs}

Plan-paid costs, consisting of claims for all pharmacy-dispensed medications, were analyzed. Participation in the diabetes 


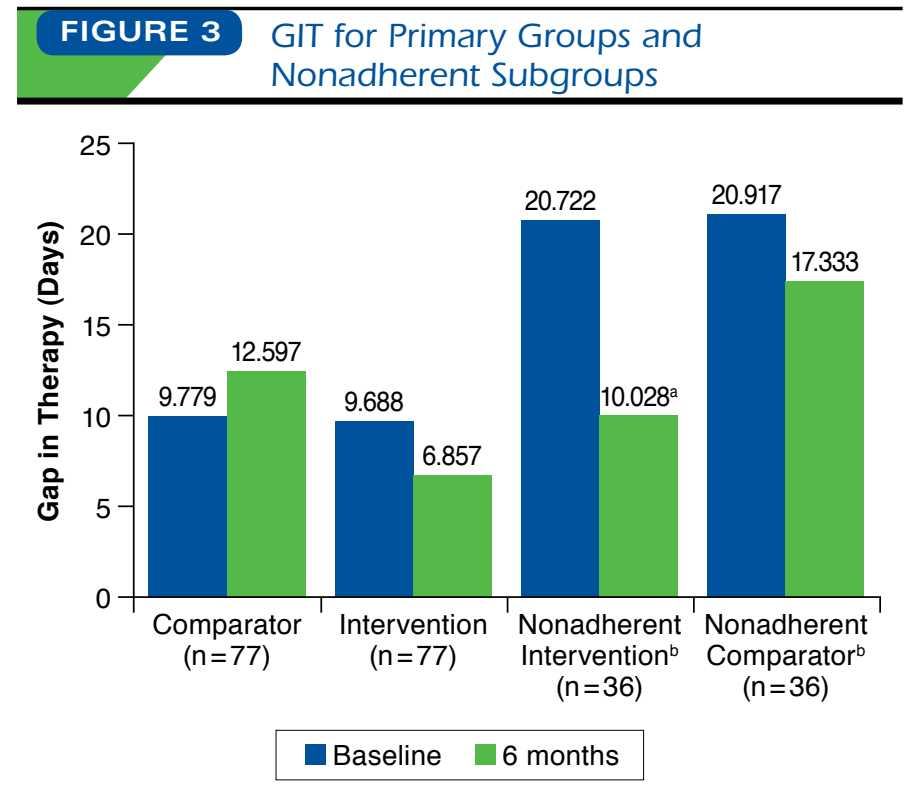

a $P<0.05$ relative to nonadherent intervention group baseline.

${ }^{b}$ Nonadherent subgroup defined as having a baseline $P D C<100 \%$.

GIT = gap in therapy; $P D C=$ proportion of days covered.

management program resulted in a significant $55 \%$ increase in the plan-paid costs per member per 6-month period $(\$ 1,991.23-\$ 3,092.74 ; P<0.05)$, as compared with a nonsignificant $17 \%$ increase in the comparator group ( $\$ 1,402.21$ $\$ 1,645.68)$. These figures are equivalent to an increase of $\$ 183.58$ (\$331.87-\$515.45) per member per month in the intervention group and an increase of $\$ 40.58$ (\$233.70-\$274.28) in the group without intervention.

\section{Discussion}

This population was found to have an average PDC at baseline of $92.9 \%$. Nearly half of the study population, 72 of 154 members, had a PDC of $100 \%$ through combined mail order and retail channels at baseline, which indicates perfect adherence. Given that the standard definition of adherence for nonspecialty maintenance medications involves meeting a PDC goal of $>80 \%,{ }^{14}$ this population would be considered remarkably adherent before intervention. This may be indicative of selection bias, since this intervention was a voluntary program. Members interested in participating in this program may have been more attentive to their health care at baseline, resulting in higher adherence than an average population.

Despite this level of adherence at baseline, the study intervention was correlated to positive trends in adherence for the overall population. The PDCs of the intervention participants trended nonsignificantly upward, while those of nonparticipants drifted downwards towards a more anticipated normal

\section{FIGURE 4 PDC by Gender Subgroups}

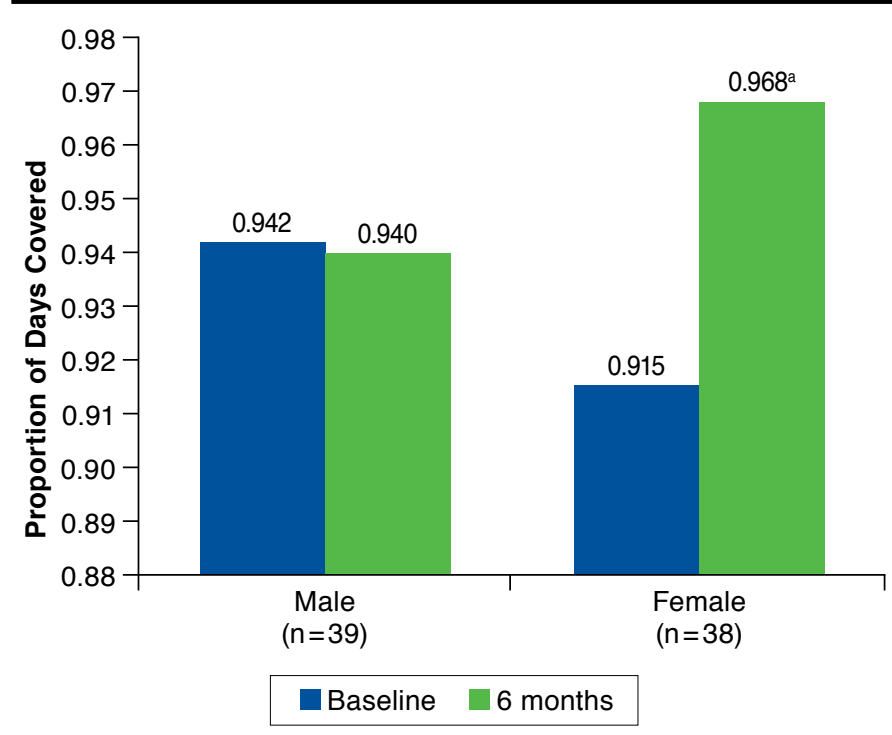

Note: Includes intervention recipients only.

a $P<0.05$ relative to female baseline.

$P D C=$ proportion of days covered.

level. The GIT trends portray a nearly identical phenomenon, with slight nonsignificant improvement after intervention compared with a normalizing effect on the comparators.

When limiting the focus to those with less than perfect adherence, the trends observed among the overall population translate to statistically significant improvements. It is also worth noting that this "nonadherent" population, per our definition, still had an average baseline PDC of $84.7 \%$, again exceeding the standard definition for adequate adherence. Although the adherence metrics, PDC and GIT, improved in the intervention and comparator populations over time, statistically significant improvements were only observed following the intervention. This supports the utility of combined VBID/ MTM programs but suggests the need to selectively target populations that are struggling to meet adherence goals.

These results indicate a disparity of response by gender. In the primary outcome, PDC, and the key secondary outcome, GIT, male participants exhibited no response to intervention. Female participants had slightly worse but statistically equivalent adherence at baseline per both metrics, but both PDC and GIT improved significantly over the 6-month observation period. This result suggests that females may be more receptive to a combined program of VBID/MTM than males. A larger, longer-term analysis may be able to reveal whether male participants are slower to adopt behavioral changes, or whether they are simply less responsive to VBID/MTM interventions. 


\section{FIGURE 5 GIT by Gender Subgroups}

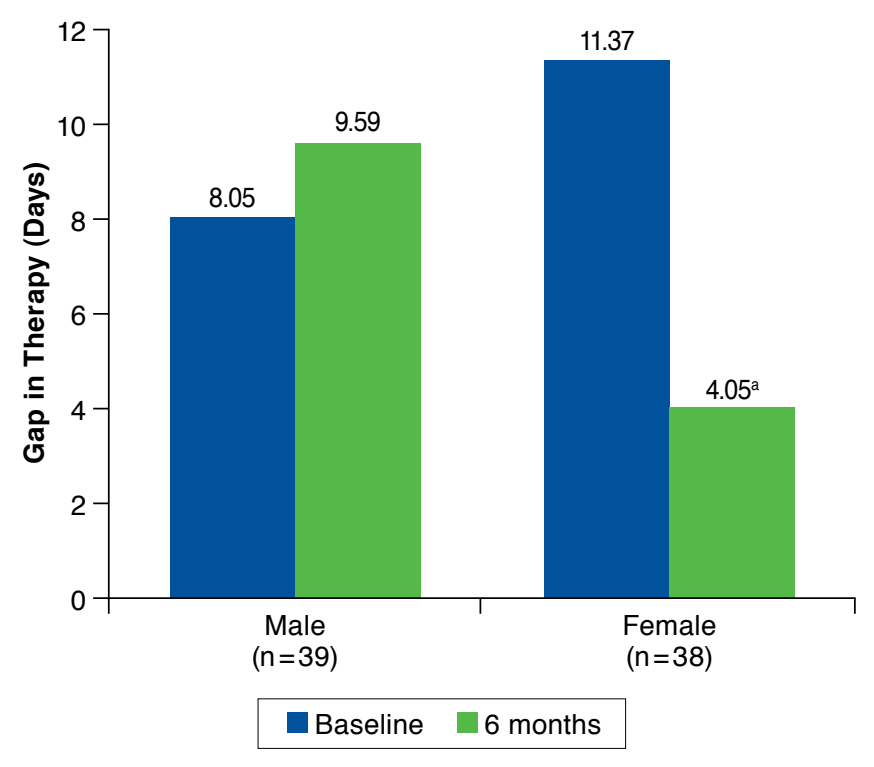

Note: Includes intervention recipients only.

a $P<0.05$ relative to female baseline.

GIT = gap in therapy.

As expected, the intervention led to increased plan-paid pharmacy costs. This increase appears to be primarily because the plan absorbed the copayment costs of the member in order to remove that barrier to adherence. In addition, this increase is likely related to increased use among participants as a direct result of increased adherence. The scope of this study did not allow for an analysis of the effect on the plan's medical spending. Since medical data were not available, the effect on clinical outcomes and potential medical savings could not be compared with the increase in pharmacy costs, so no conclusion regarding the cost-effectiveness of intervention may be made from this study.

\section{Limitations}

The primary limitation of this study is the small sample size. The intervention has yet to receive widespread participation, limiting the analysis to a small number of participants. As such, it may be difficult to generalize these results to a broader population.

The results of this analysis are further limited by selection bias. Because of the voluntary nature of this intervention, members who chose to participate in the program may have been more proactive in the management of their diabetes at baseline. These members may have therefore had less improvement potential than members who declined the intervention.
This bias may be evidenced by the elevated baseline adherence metrics in this population.

A full analysis of the benefits of this intervention was further limited by a lack of access to medical data. While increased adherence can be tied to improved diabetes outcomes, ${ }^{6}$ specific data on the effect of intervention on outcomes such as Alc, blood pressure, and lipid management; emergency department visits; and hospitalizations would increase the value of this study. Additionally, the effect of intervention on medical costs for members relative to direct and indirect program costs would be of significant value to health plans, pharmacy benefit managers, payers, and other stakeholders.

The length of this study also limits utility. The 6-month postintervention window shows the immediate effect of the program but does not provide information on the durability of response. A durable increase in adherence would be necessary to realize true health outcome and health care cost benefits. Additionally, the duration affects the robustness of adherence measurements, with limited data being more susceptible to aberrations

\section{Conclusions}

Although the analysis of the overall population did not indicate statistically significant improvements to adherence, the improvement trends and subpopulation analyses demonstrated that combined VBID/MTM programs may positively influence member behavior in the setting of an employer group. Secondary analyses provided evidence that VBID/MTM programs may be particularly valuable when applied to members with lower adherence rates and female members; however, more research involving longer durations and greater patient numbers is needed. Additionally, further research including health outcomes and medical spending would provide substantial value to the current body of VBID/MTM evidence.

\section{Authors}

ALEX PEASLEE, PharmD; MARLEEN WICKIZER, PharmD, AE-C; and JULIE OLSON, DNP, MS, RN, CQIA, CBE, Navitus Health Solutions, Madison, Wisconsin. ROBERT TOPP, PhD, RN, University of San Diego Hahn School of Nursing and Health Science, San Diego, California.

AUTHOR CORRESPONDENCE: Alex Peaslee, PharmD, Clinical Staff Pharmacist, Navitus Health Solutions, 2601 W. Beltline Hwy., Ste. 600, Madison, WI 53713. Tel.: 608.729.1625;

Fax:608.729.2625; E-mail: Alex.Peaslee@Navitus.com. 


\section{DISCLOSURES}

Funding for this project was provided by Navitus Health Solutions. Peaslee, Wickizer, and Olson are employed by Navitus Health Solutions. Peaslee is a clinical staff pharmacist working in Formulary Services and a former PGY-1 Managed Care Clinical Pharmacy Resident at this location. Wickizer is the Associate Manager of Clinical Programs and Residency Programs. Olson is the Director of Clinical Programs and Product Development. Topp is the Patricia A. Chin Nursing Research Endowed Professor at the Hahn School of Nursing and Health Science at the University of San Diego specializing in statistics. Topp received consulting fees from Navitus Health Solutions for work on this project.

Study concept and design were contributed by Peaslee, Wickizer, and Olson, with assistance from Topp. Peaslee took the lead in data collection, with assistance from Wickizer, and data interpretation was performed by Peaslee, Topp, Wickizer, and Olson. The manuscript was written primarily by Peaslee, with assistance from the other authors, and revised by Topp, Wickizer, and Olson, assisted by Peaslee.

\section{REFERENCES}

1. Zeber JE, Manias E, Williams AF, et al. A systematic literature review of psychosocial and behavioral factors associated with initial medication adherence: a report of the ISPOR Medication Adherence \& Persistence Special Interest Group. Value Health. 2013;16(5):891-900.

2. Chernew ME, Shah MR, Wegh A, et al. Impact of decreasing copayments on medication adherence within a disease management environment. Health Aff (Millwood). 2008;27(1)103-12.

3. Kennedy J, Tuleu I, Mackay K. Unfilled prescriptions of medicare beneficiaries: prevalence, reasons, and types of medicines prescribed. J Manag Care Pharm. 2008;14(6):553-60. Available at: http://www.jmcp.org/doi/ abs/10.18553/jmcp.2008.14.6.553.

4. Chernew ME, Rosen AB, Fendrick AM. Value-based insurance design. Health Aff (Millwood). 2007;26(2):w195-203.
5. Lee JL, Maciejewski ML, Raju SS, et al. Value-based insurance design: quality improvement but no cost savings. Health Aff (Millwood). 2013;32(7):1251-57.

6. Elliott DJ, Robinson EJ, Anthony KB, Stillman PL. Patient-centered outcomes of a value-based insurance design program for patients with diabetes. Popul Health Manag. 2013;16(2):99-106.

7. Zimmerman A. The changing role of pharmacy benefit administration in managing chronic conditions. Benefits Q. 2014;30(1):13-18.

8. van Boven JF, Stuurman-Bieze AG, Hiddink EG, Postma MJ, Vegter S. Medication monitoring and optimization: a targeted pharmacist program for effective and cost-effective improvement of chronic therapy adherence. J Manag Care Spec Pharm. 2014;20(8):786-92. Available at: http://www.jmcp. org/doi/10.18553/jmcp.2014.20.8.786.

9. Cranor CW, Bunting BA, Christensen DB. The Asheville Project: longterm clinical and economic outcomes of a community pharmacy diabetes care program. J Am Pharm Assoc. 2003;43(2):173-84.

10. Han E, Suh DC, Lee SM, Jang S. The impact of medication adherence on health outcomes for chronic metabolic diseases: a retrospective cohort study. Res Social Adm Pharm. 2014;10(6):e87-98.

11. Chernew ME, Juster IA, Shah M, et al. Evidence that value-based insurance can be effective. Health Aff (Millwood). 2010;29(3):530-36.

12. Congressional Budget Office. Offsetting effects of prescription drug use on Medicare's spending for medical services. November 29, 2012. Available at: https://www.cbo.gov/publication/4374l. Accessed September 10, 2016.

13. Wertz D, Hou L, DeVries A, et al. Clinical and economic outcomes of the Cincinnati Pharmacy Coaching Program for diabetes and hypertension. Manag Care. 2012;21(3):44-54.

14. Ho PM, Bryson CL, Rumsfeld JS. Medication adherence: its importance in cardiovascular outcomes. Circulation. 2009;119(23):3028-35. 